Supporting Information

\title{
Functionalizing Polystyrene with $N$-alicyclic Piperidine-Based Cations via Friedel-Crafts Alkylation for Highly Alkali-Stable Anion-Exchange Membranes
}

Joel S. Olsson, Thanh Huong Pham and Patric Jannasch*

Polymer \& Materials Chemistry, Department of Chemistry, Lund University,

P.O. Box 124, SE-221 00, Lund, Sweden

*E-mail: patric.jannasch@chem.lu.se 


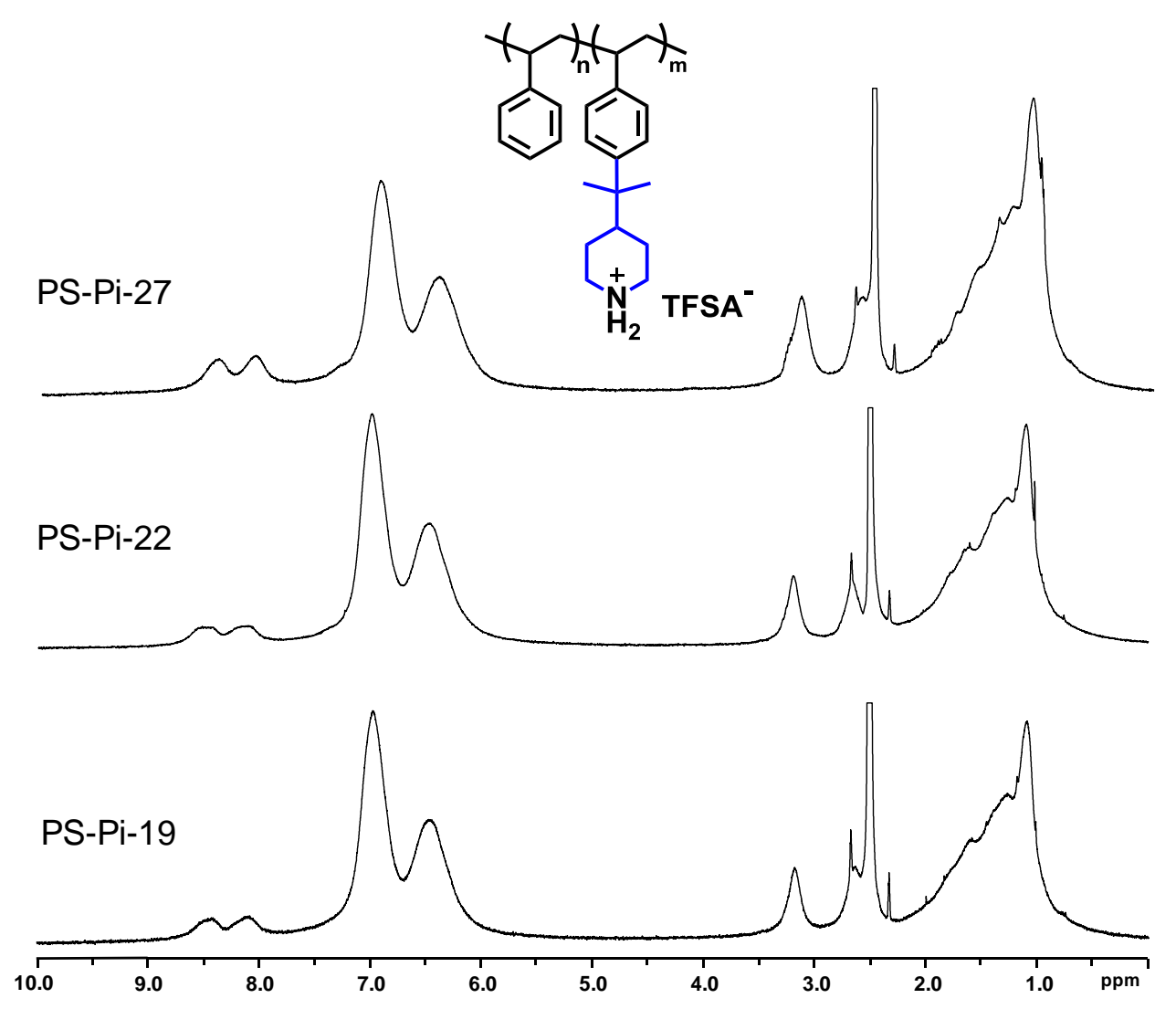

Figure S1. ${ }^{1} \mathrm{H}$ NMR spectra of precursor polymers in DMSO- $d_{6}$.

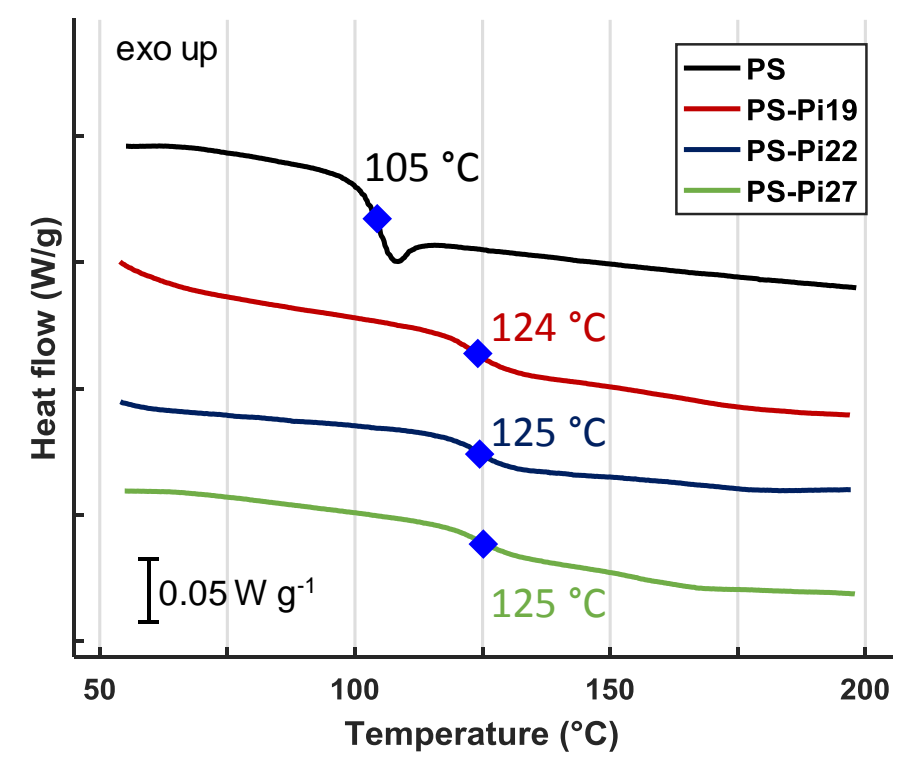

Figure S2. DSC traces of neat polystyrene and the PS-Pi19 and PS-Pi27 precursors with the glass transitions indicated. Data were recorded at $10{ }^{\circ} \mathrm{C} \mathrm{min}^{-1}$ under nitrogen atmosphere. 


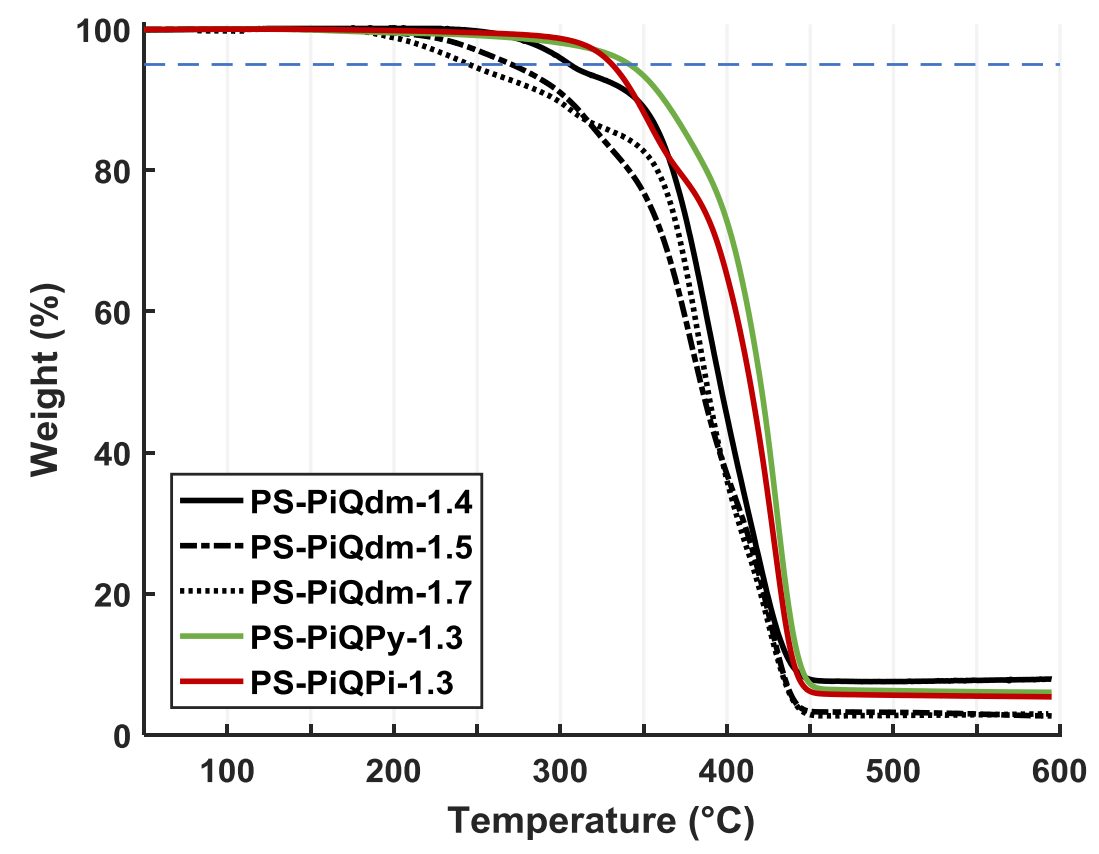

Figure S3. TGA traces of the AEMs in the $\mathrm{Br}^{-}$form recorded at $10{ }^{\circ} \mathrm{C} \mathrm{min}{ }^{-1}$ under nitrogen atmosphere.

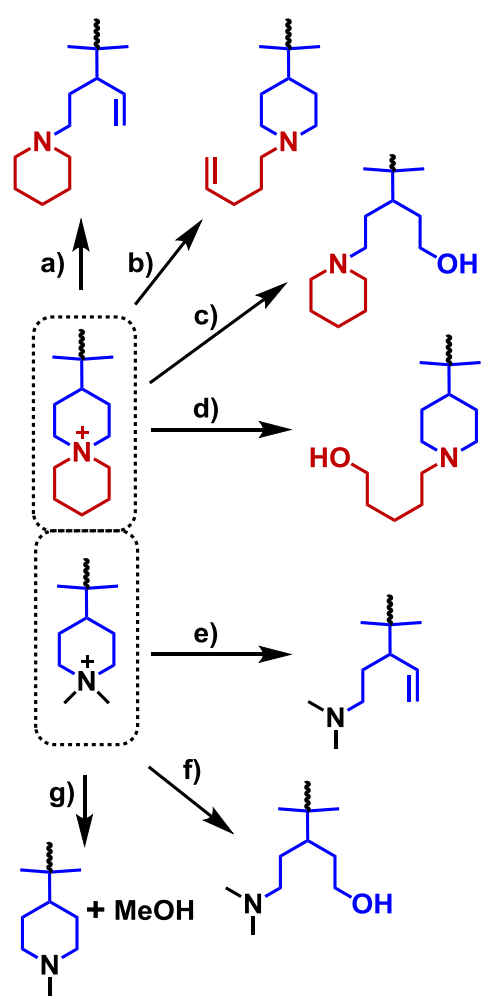

Scheme S1. Degradation pathways of mono- and $N$ spirocyclic cations in the presence of $\mathrm{OH}^{-}$leading to loss of the charged center. Hofmann $\beta$-elimination in the upper ring $(\mathrm{a}, \mathrm{e})$ and in the pendant ring (b), nucleophilic attack on an $\alpha$-carbon in the upper ring (c, f) and in the pendant ring (d) all leading to ring-opening. In addition, the monocyclic dimethylpiperidinium cation can degrade via methyl substitution $(\mathrm{g})$. 


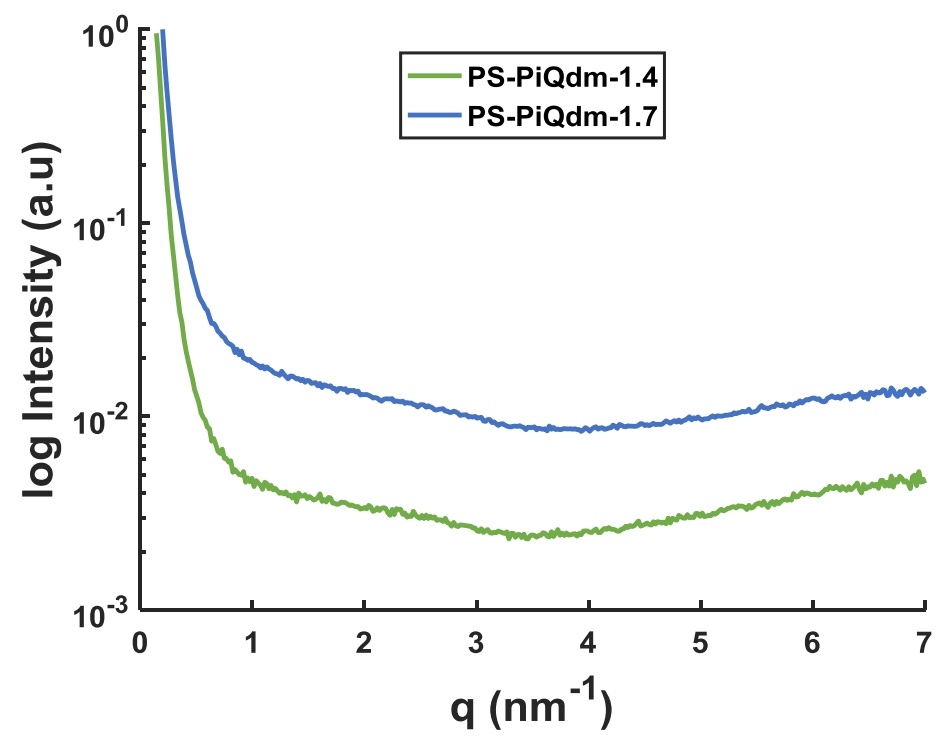

Figure S4. SAXS profiles of dry PS-PiQdm-1.4 and -1.7 AEMs in the $\mathrm{Br}^{-}$form.

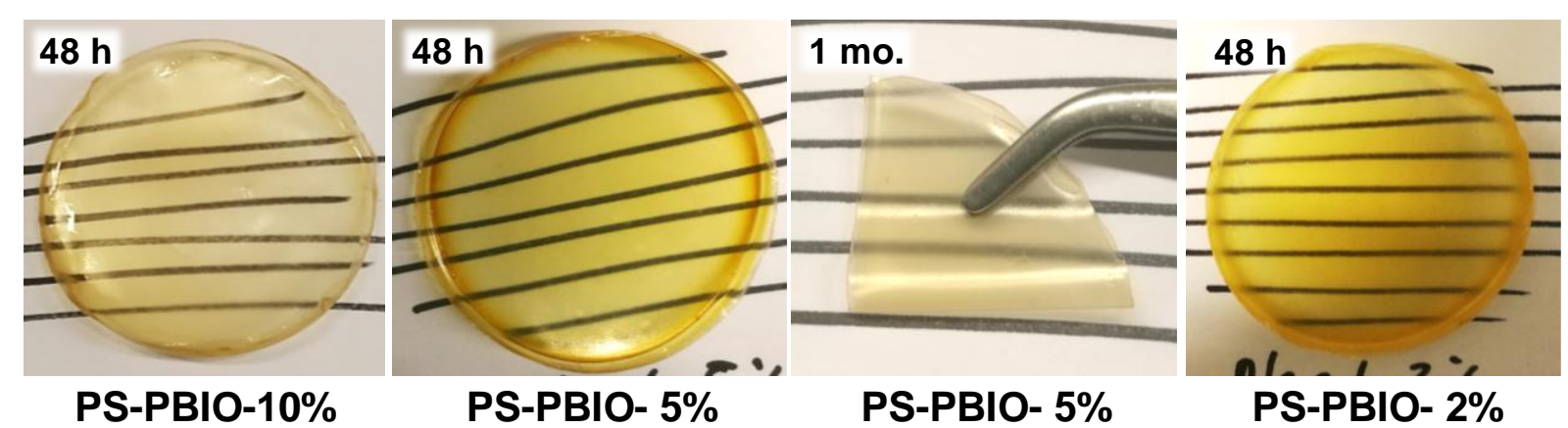

Figure S5. Photographs (enlarged) of the AEMs displayed in Figure 6. 

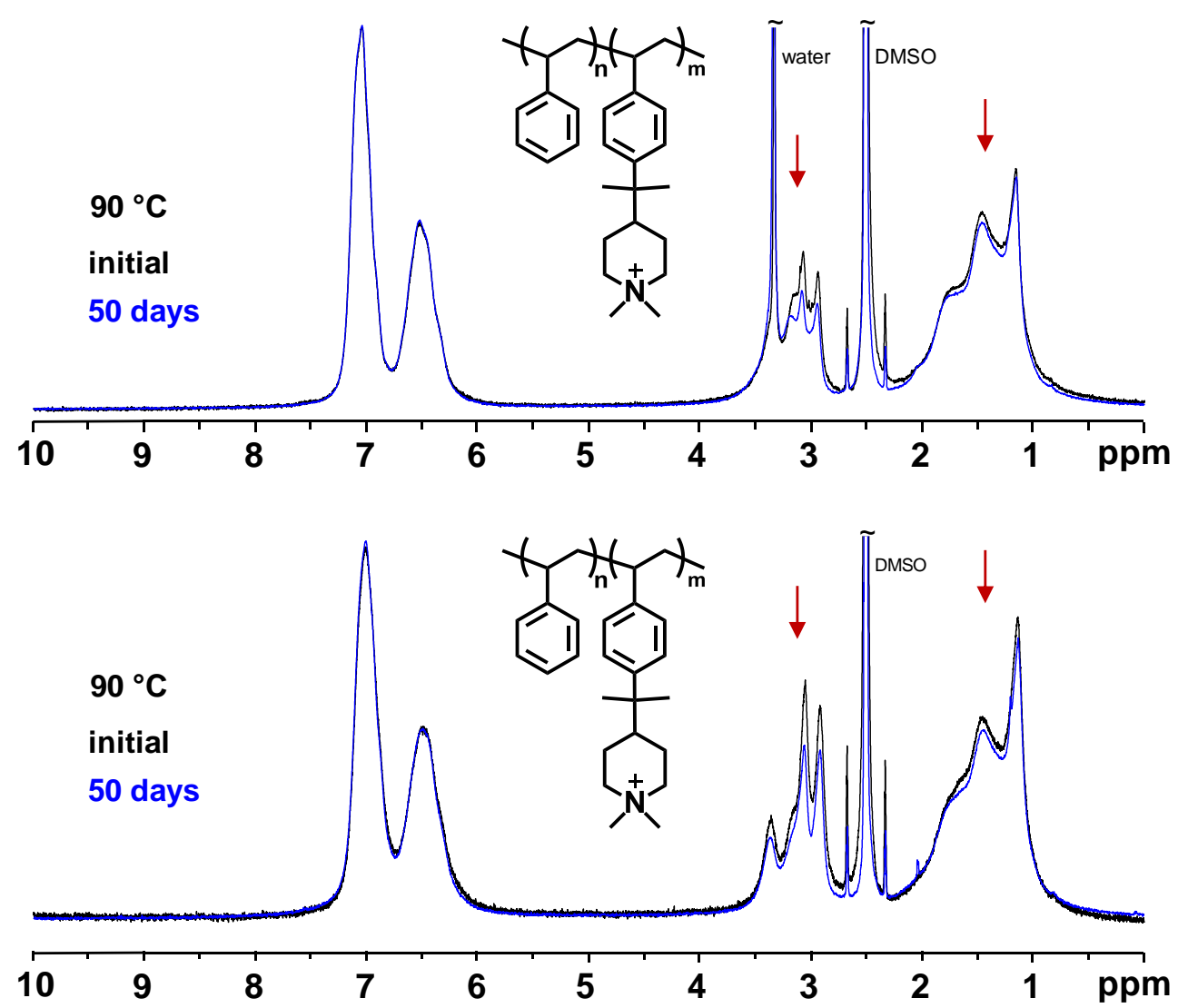

Figure S6. Overlaid ${ }^{1} \mathrm{H}$ NMR spectra of PS-PiQdm-1.4 in DMSO- $d_{6}$ before and after storage in $2 \mathrm{M}$ aq. $\mathrm{NaOH}$ at $90{ }^{\circ} \mathrm{C}$ illustrating the intensity decrease of the signals in region III and the accompanying decrease between 1 and $2 \mathrm{ppm}$. At this magnification, no other changes can be distinguished. Above no TFA added, below with 5-10 vol\% TFA added to the DMSO- $d_{6}$ solution. 


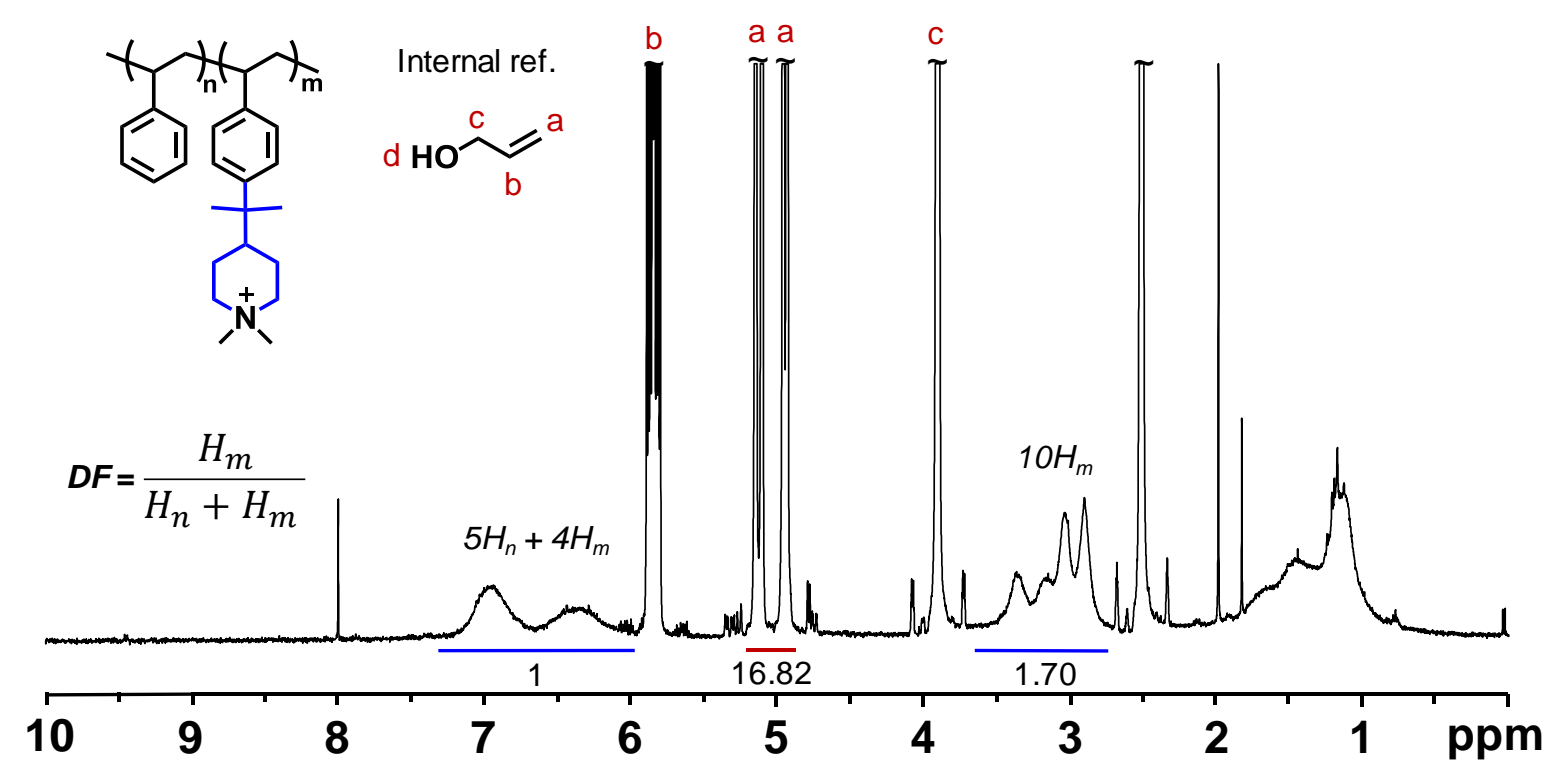

Figure S7. ${ }^{1} \mathrm{H}$ NMR spectra of cationic PS found dissolved in the alkaline solution after immersion of PS-PiQdm-1.7 (DF $=27 \%)$ during 10 days in $0.1 \mathrm{M}$ aq. $\mathrm{NaOH}$ at $120{ }^{\circ} \mathrm{C}$. In order to calculate the mass of the leeched polymer an internal standard (allyl alcohol) was added to the NMR solution. The average DF of the leeched polymer was calculated to be approximately $73 \%$ using the equation shown in the figure, corresponding to an IEC of 3.2 mequiv. $\mathrm{g}^{-1}$. The amount of leeched polymer was approximately $3 \mathrm{wt} \%$ of the whole sample $(12.46 \mathrm{mg})$, corresponding to a loss of $\sim 8 \%$ of the functional groups from the AEM.

The controlled degradation test was undertaken in $0.1 \mathrm{M}$ aq. $\mathrm{NaOH}$ at $120{ }^{\circ} \mathrm{C}$. After 10 days, the sample was taken out and the water in the liquid phase was evaporated slowly at $40{ }^{\circ} \mathrm{C}$ on a petri dish placed in a fume hood. The solid residues, salt and a polymer film, was transferred to a vial and dissolved in DMSO- $d_{6}$. To ensure quantitative transfer, the Petri dish was also washed with DMSO- $d_{6}$. The liquid phase (DMSO solution) was transferred with a Pasteur pipette to an NMR tube. TFA and $3.8 \mathrm{mg}$ of allyl alcohol was added to NMR tube using a micropipette before analysis. In parallel, the AEM samples were washed and dried at $50{ }^{\circ} \mathrm{C}$ in vacuum over 5 days before weighing. The use of allyl alcohol ( $a, b$ and $c$ ) as an internal standard enabled estimation of the mass of the leeched polymer. 
a)
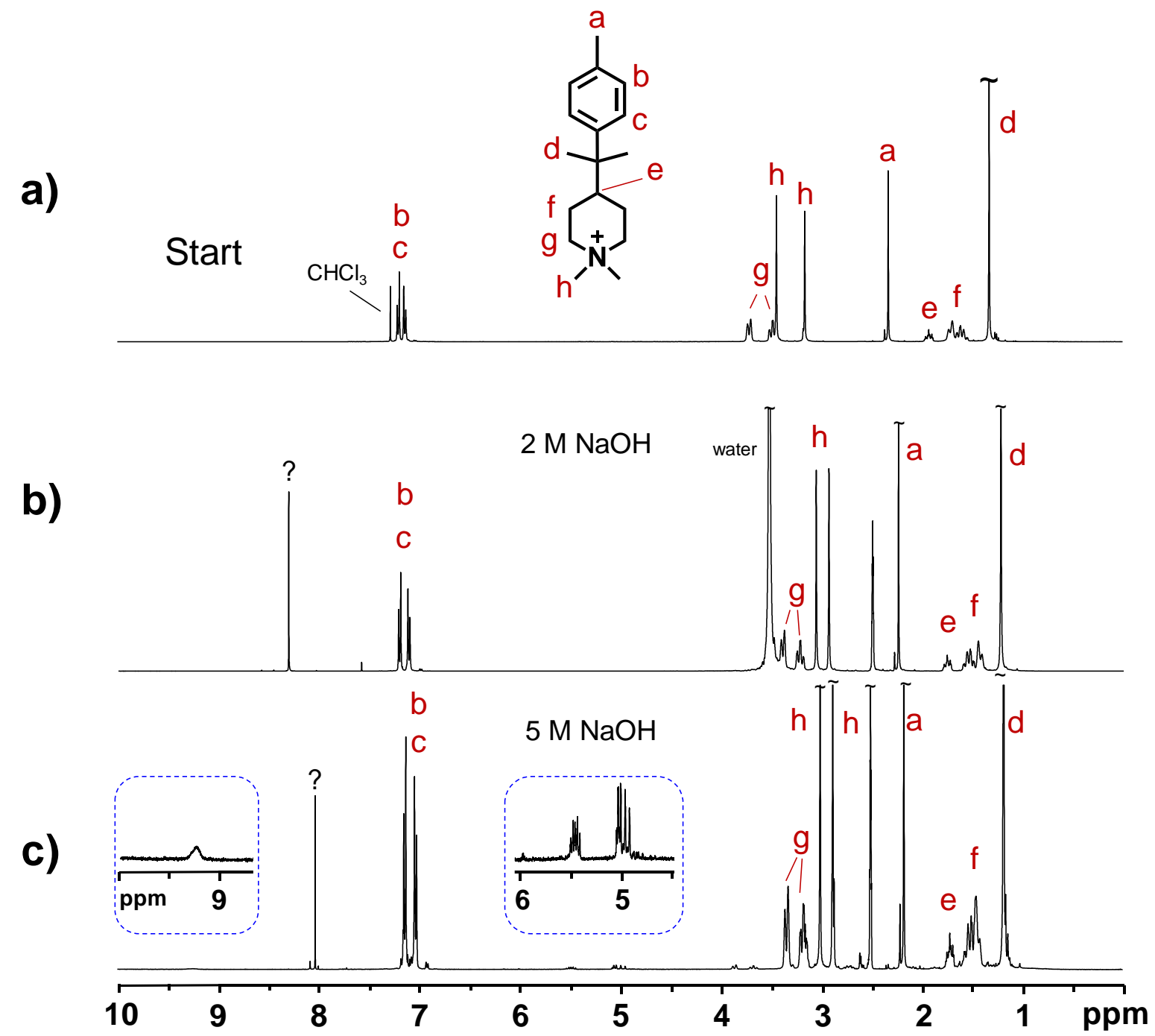

Figure S8. ${ }^{1} \mathrm{H}$ NMR spectra of the model compound 2-(dimethyl piperidinium-4-yl)propan-2-tolyl before (a) and after alkaline treatment in $2 \mathrm{M}$ (b) and $5 \mathrm{M}$ (c) aq. $\mathrm{NaOH}$ at $120{ }^{\circ} \mathrm{C}$ during 14 days. Proton signals have been assigned. 
PS-PBIO-5\%
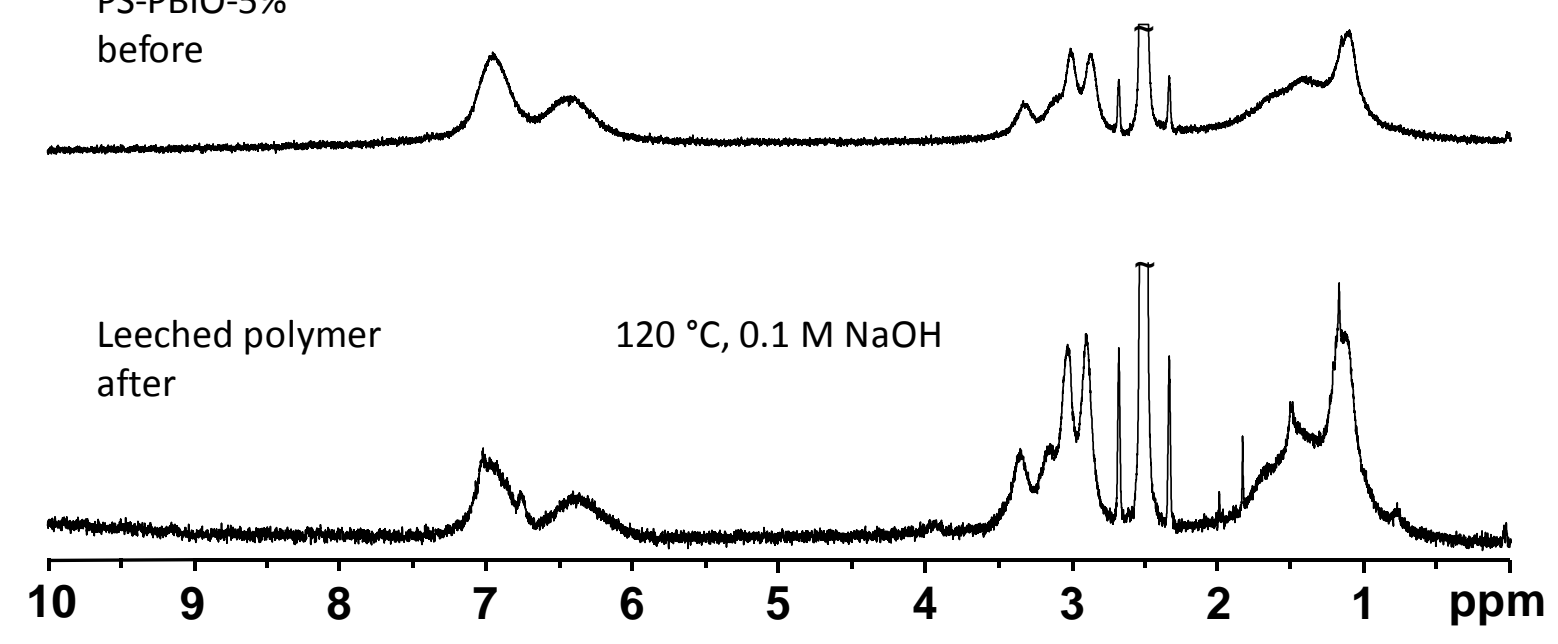

Figure S9. ${ }^{1} \mathrm{H}$ NMR spectra of a) PS-PBIO-5\% in DMSO- $d_{6}$ and b) cationic PS found dissolved in the alkaline solution after immersion of the blend AEM PS-PBIO-5\% during 10 days in $0.1 \mathrm{M}$ aq. $\mathrm{NaOH}$ at $120^{\circ} \mathrm{C}$. TFA was used as a co-solvent in both spectra. As can be seen, the overlapping signals at 3 ppm (b), which originate from protons in $\alpha$-position to the charged center, are significantly larger than corresponding signals before the test (a), indicating that the leeched polymer had a higher IEC than the average of the PS used for blending. 